\title{
El debate sobre los territorios inteligentes: el caso del área metropolitana de Sevilla*
}

\author{
INMACULADA CARAVACA BARROSO** Y ANTONIO GARCÍA GARCÍA*** \\ ** Departamento de Geografía Humana, Universidad de Sevilla. \\ *** Departamento de Geografía, Historia y Filosofía, Universidad Pablo de Olavide, Sevilla.
}

\begin{abstract}
The fast and deep transformations experienced by the societies, the economies and the spatial patterns during the last decades, demands that researchers make new interpretations and public managers come up with new answers to face the current problems and challenges. In this general context, the purpose of this article is to participate of the debate about the concept of intelligent territory, combining economicist approaches with others ones related to the idea of integrated territorial development. In order to achieve this aim the evolution experienced by the metropolitan area of Sevilla will be analyzed, using this double approach.
\end{abstract}

KEY WORDS: intelligent territory, socioeconomic transformations, metropolitan area of Seville.

RESUMEN Los cambios profundos y rápidos que están experimentando las sociedades, las economías y las lógicas territoriales durante las últimas décadas, demandan nuevas lecturas e interpretaciones por parte de los investigadores y nuevas respuestas de los responsables públicos para poder hacer frente a los problemas y retos que ahora se perfilan. En este contexto general de referencia, el objetivo de este artículo es participar en el debate sobre el concepto de territorio inteligente, aunando la perspectiva economicista con aquella otra más ligada a la idea de desarrollo territorial integrado. Ello, a través del análisis de la evolución experimentada por el área metropolitana de Sevilla, realizado con esta doble mirada.

PALABRAS CLAVES: territorio inteligente, transformaciones socioeconómicas, área metropolitana de Sevilla.

\footnotetext{
Este artículo forma parte del Proyecto de I+D del Ministerio español de Educación y Ciencia SEJ 2006-14277C04-03.

Recibido el 18 de julio de 2008, aprobado el 5 de junio de 2009.

Correspondencia: Inmaculada Caravaca Barroso, Departamento de Geografía Humana, Universidad de Sevilla, C/ María de Padilla s/n, 41004, Sevilla, España. E-mail: caravaca@us.es. Antonio García García, Departamento de Geografía, Historia y Filosofía, Universidad Pablo de Olavide, Ctra. de Utrera Km. 1, 41013, Sevilla, España. E-mail: agargar1@upo.es.
} 
Las fuerzas ciegas de la urbanización, fluyendo a lo largo de las lineas de menor resistencia, no muestran ninguna capacidad de crear un modelo urbano e industrial que sea estable, sostenible y renovable. Por el contrario, según aumenta la congestión y prosigue la expansión de la ciudad, tanto el paisaje urbano como el rural se desfiguran y se degradan, al tiempo que las inútiles inversiones para solucionar la congestión, como la construcción de nuevas autopistas o la utilización de recursos hidricos más distantes, aumentan las cargas económicas y sólo sirven para promover más ruina y desorden del que intentan paliar.

(Historia Natural de la Urbanización, Lewis Mumford, 1956)

\section{¿Qué se entiende por territorio inteligente?}

Las aceleradas y profundas transformaciones que están experimentando las sociedades, las actividades económicas y los territorios durante las últimas décadas, están dando lugar a toda una serie de lecturas e interpretaciones por parte de los investigadores y hacen necesarias nuevas respuestas de los responsables públicos para hacer frente a los problemas y retos que se están generando.

Especial importancia adquieren dichas transformaciones en las aglomeraciones metropolitanas que si, por una parte, son consideradas territorios que ganan al actuar como centros neurálgicos de poder y principales nodos de articulación del espacio de redes (Benko y Lipietz, 1994; Veltz, 1996; Caravaca, I. 1998), por otra, están especialmente afectadas por graves problemas y disfuncionalidades que las hacen social y ambientalmente insostenibles (Fernández Durán, 1993; Acselrad, 1999; Cano, A., 2004). Se trata, pues, de territorios muy cambiantes y complejos en los que los impactos de los grandes procesos en curso están siendo especialmente profundos y significativos. El esquema de la figura 1 pretende sintetizarlos y relacionarlos con toda una serie de categorías conceptuales que se vienen utilizando por investigadores de distintas disciplinas y que, aunque pueden resultar expresivas, también parecen a veces algo confusas y son "un síntoma de las dificultades para precisar el sentido, las claves explicativas y la dimensión de tales cambios" (Méndez- Rodríguez Moya, 2007, p. 106). 
Figura 1

PRINCIPALES TRANSFORMACIONES METROPOLITANAS Y NUEVAS CATEGORÍAS CONCEPTUALES.

\begin{tabular}{|c|c|c|}
\hline Procesos & Transformaciones & Nuevas categorias conceptuales \\
\hline $\begin{array}{l}\text { Revolución } \\
\text { tecnológica }\end{array}$ & $\begin{array}{l}\text { *Consolidación de la sociedad de la } \\
\text { información y del conocimiento } \\
\text { *Creciente importancia de las } \\
\text { innovaciones }\end{array}$ & $\begin{array}{l}\text { *Ciudad informacional (Castells) } \\
\text { *Ciudad digital (Masuda) } \\
\text { *Ciberciudad (Graham) } \\
\text { *Ciudad innovadora (Méndez) } \\
\text { *Ciudad creativa (Landry, Florida) } \\
\text { *Ciudad que aprende (Morgan) } \\
\text { *Ciudad inteligente (Komninos) }\end{array}$ \\
\hline $\begin{array}{l}\text { Globalización } \\
\text { económica }\end{array}$ & $\begin{array}{l}\text { *Espacio de redes como lógica } \\
\text { dominante } \\
{ }^{*} \text { Contraposición espacio de } \\
\text { redes/espacio de lugares } \\
{ }^{*} \text { Cambios en la relación } \\
\text { espacio/tiempo }\end{array}$ & $\begin{array}{l}\text { *Ciudad global (Sassen) } \\
\text { *Ciudad planetaria (Reich) } \\
\text { *Metrópolis transnacional (Cordeiro) } \\
\text { *Ciudad mundial (Knox, Taylor) } \\
\text { *Ciudad globalizada (Moulaert, } \\
\text { Rodríguez, Swyngedow) }\end{array}$ \\
\hline $\begin{array}{l}\text { Reestructuración } \\
\text { productiva y nueva } \\
\text { fase capitalista }\end{array}$ & $\begin{array}{l}\text { *Terciarización económica e } \\
\text { industrial } \\
\text { *Especialización } \\
\text { flexible/postfordismo }\end{array}$ & $\begin{array}{l}\text { *Ciudad postindustrial (Bell) } \\
\text { *Ciudad terciaria (Sánchez) }\end{array}$ \\
\hline $\begin{array}{c}\text { Crecimiento de los } \\
\text { territorios } \\
\text { metropolitanos }\end{array}$ & $\begin{array}{l}\text { * Procesos de difusión territorial } \\
\text { de las actividades económicas y la } \\
\text { población } \\
\text { *Ampliación área economías externas } \\
\text { de aglomeración y urbanización } \\
\text { *Dinamismo de espacios periurbanos }\end{array}$ & $\begin{array}{l}\text { *Megaciudad (Bronger) } \\
\text { *Ciudad-región (Scott, Friedman) } \\
\text { *Ciudad infinita (Bonomi-Abruzzese) } \\
\text { *Metropolización expandida (De Mattos) }\end{array}$ \\
\hline $\begin{array}{l}\text { Reorganización } \\
\text { territorial } \\
\text { metropolitana }\end{array}$ & $\begin{array}{l}\text { *Formación de subcentros } \\
\text { suburbanos/ villas emergentes } \\
\text { *Concentración descentralizada } \\
\text { *Nuevos espacios de producción, } \\
\text { ocio y consumo } \\
\text { *Discontinuidad física/integración } \\
\text { funcional }\end{array}$ & $\begin{array}{l}\text { *Ciudad dispersa (Hayes) } \\
\text { *Ciudad difusa (Indovina, Monclus) } \\
\text { *Ciudad interrumpida (Delgado) } \\
\text { *Edge cities (Garreau) } \\
\text { *Ciudad reticulada (Dematteis) } \\
\text { *Metrópolis policéntricas (Hall, Pain) } \\
\text { *Metápolis (Ascher). }\end{array}$ \\
\hline $\begin{array}{l}\text { Fragmentación } \\
\text { socioeconómica de } \\
\text { los espacios } \\
\text { urbanos }\end{array}$ & $\begin{array}{l}\text { *Áreas urbanas dinámicas frente a } \\
\text { otras marginadas o en declive } \\
\text { *Creciente proceso de zonificación } \\
\text { socioeconómica }\end{array}$ & $\begin{array}{l}\text { *Ciudad dual (Muxi) } \\
\text { *Ciudad desigual (Hamnet). } \\
\text { *Postmetrópolis (Soja) }\end{array}$ \\
\hline
\end{tabular}

Fuente: Elaboración propia

Entre las múltiples tensiones y contradicciones a que las sociedades actuales deben hacer frente adquiere especial importancia la forma en que se articulan los distintos ámbitos territoriales al espacio global de los flujos y las redes, existiendo un cierto consenso en que la capacidad innovadora, que ha permitido la consolidación de la sociedad de la información y del conocimiento, es la que en mayor medida condiciona la forma de inserción de empresas y territorios en un espacio mundial, desequilibrado y muy cambiante, en el que se contraponen áreas innovadoras, capaces de responder con éxito a los nuevos retos, a aquéllas otras marginadas o incluso excluidas porque, además de razones estructurales, su falta de espíritu innovador les 
impide reaccionar para adaptarse a la lógica de las redes y a los incesantes cambios económicos, sociales, laborales, institucionales y territoriales que se vienen produciendo.

Frente a la búsqueda de respuestas innovadoras capaces de mejorar la competitividad de las empresas y los territorios, se valora también cada vez más la capacidad tanto de las sociedades - para incorporar conocimiento y ser creativas en la búsqueda de formas que permitan poner en valor todos los recursos-como de las instituciones — para aplicar políticas y formas de gestión comprometidas y responsables que tengan como objetivo prioritario mejorar la calidad de vida de todos las personas- E . En este sentido, como señala Méndez, "frente a visiones de la innovación que muestran un acusado sesgo tecnológico y económico, preocupadas ante todo por la mejora competitiva de las empresas y la inserción de las ciudades en los mercados mundiales, aquí se plantea la necesidad de lo que Bradford (2003) califica como innovación comunitaria, destinada también a lograr mejoras en la gestión de los asuntos urbanos, una profundización en la democracia participativa y una más efectiva satisfacción de las necesidades de la población" (Méndez, 2009, p. 28). La innovación, entendida, pues, tanto desde la perspectiva económica como desde la vertiente social e institucional, se convierte de este modo en un importante factor que condiciona no sólo el dinamismo económico sino también y sobre todo el desarrollo territorial.

No es de extrañar, por consiguiente, que en los últimos años la innovación se haya erigido en el punto de confluencia de diferentes corrientes epistemológicas y disciplinas científicas preocupadas por las relaciones entre las actividades económicas y los territorios (Feldman, 1994; Maillat-Kebir, 1998; Cating-Lacour-Lung, 2001; Camagni-Maillat, 2006), haciéndose en algunos casos una lectura muy abierta de la innovación, al entenderla no sólo desde la perspectiva empresarial y económica sino también desde la social e institucional (McKimmonCumbers-Chapman, 2002; Moulart-Sekia, 2003; Méndez, 2002 y 2007; Albertos-CaravacaMéndez-Sánchez, 2004; Moulaert y Nussbaumer, 2005). Junto a esta pluralidad de planteamientos, se observa también la existencia de una cierta confusión en las formas en que se utiliza el concepto de innovación, en bastantes ocasiones excesivamente vinculado a las nuevas tecnologías; asimismo, la proliferación de ciertas expresiones, como la de territorio inteligente, para hacer referencia a aquellos ámbitos en los que adquiere mayor protagonismo la incorporación de conocimiento (Florida, 1995; Morgan, 1997; Jambes, 2001; Komninos, 2002; Martínez Fernández, 2004).

Si bien es cierto que la categoría conceptual de territorio inteligente se está utilizando más generalizadamente en relación con la capacidad competitiva de algunos ámbitos, cabe también otra forma muy distinta de entenderla e interpretarla. Así, y tal como apunta Marina (1993), sólo deberían merecer la categoría de inteligentes aquellos territorios capaces de generar o incorporar los conocimientos necesarios para poner en valor de forma eficiente y racional sus propios recursos para así contribuir a mejorar la calidad de vida de la sociedad que los habita y a estimular el desarrollo personal de todos los ciudadanos. En este último sentido se ha utilizado en algunas publicaciones (Caravaca-González-Silva, 2005; García-Ojeda-Torres, 2008).

Desde esta segunda perspectiva se debe incidir en la importancia de conceptos subjetivos como el arraigo, la identidad o el reconocimiento, cuestiones éstas que cualifican y dan sentido 
pleno a la idea de sostenibilidad ambiental y desarrollo territorial y que ponen de relevancia la urgencia de tratar aspectos como el patrimonio, el paisaje o la diversidad de usos del territorio (Carmona-Heath-Tiesdell, 2001). Se entiende así que desde esta segunda perspectiva un territorio inteligente, además del producto de una gestión eficiente de sus recursos, también requiere su uso inteligente por parte de los ciudadanos que deben aprovechar las oportunidades creadas, al mismo tiempo que generar o reclamar otras.

En este contexto general de referencia y teniendo en cuenta estas dos formas de acercarse al conocimiento de los territorios inteligentes, el objetivo de este artículo es participar en el debate sobre el concepto de territorio inteligente, aunando la perspectiva economicista con aquella otra más ligada a la idea de desarrollo territorial integrado. Ello, a través del análisis de la evolución experimentada por la aglomeración metropolitana de Sevilla, realizado con esta doble mirada.

No resulta baladí la utilización de un ámbito territorial específico como el que se presenta, ya que, además de la bibliografía de carácter teórico y general que nos ayuda a profundizar en el significado de la innovación, la sociedad del conocimiento y el carácter más o menos inteligente de los territorios, permite utilizar también algunos trabajos de carácter empírico, así como una serie de indicadores que nos permiten analizar el comportamiento de esta aglomeración desde la perspectiva que aquí interesa.

\section{Caracterización territorial y proceso de conformación de la aglomeración metropolita- na de Sevilla}

Ocupando una superficie de $4.900 \mathrm{Km} 2$, la aglomeración metropolitana de Sevilla está compuesta por 46 municipios e incluye a una población de 1.450.214 habitantes (Padrón Municipal de Habitantes, 2007). Por su tamaño se trata, pues, de una aglomeración de tamaño medio, que ocupa la cuarta posición en el sistema urbano español, detrás de Madrid, Barcelona y Valencia.

Desde el punto de vista físico el Guadalquivir es su principal referente, puesto que este río y sus afluentes condicionan la orografía de su emplazamiento, caracterizada por una morfología llana a excepción de las plataformas del Aljarafe y Los Alcores.

En consonancia con las tendencias generales presentes en este tipo de ámbitos, el espacio sevillano considerado metropolitano ha experimentado cambios significativos durante las últimas décadas; la dinamización económica observada desde finales de los años ochenta, unida al impulso dado por las inversiones públicas relacionadas en parte con la Exposición Universal de 1992, contribuyeron decisivamente al proceso de construcción metropolitana. Los movimientos centrípetos que fueron característicos en los años sesenta y setenta, se invirtieron a fines de los ochenta, iniciándose una fase en la que, en consonancia con lo ocurrido en las principales aglomeraciones metropolitanas del entorno, predominan ya los flujos de carácter centrífugo, produciéndose, a la vez, una ralentización en el crecimiento poblacional del conjunto del área, mientras aumenta en extensión el territorio metropolitano (Figura 2). 
En este último sentido, se ha impuesto el modelo de baja densidad de la ciudad difusa de viviendas adosadas — avalado por una presunta consecución de calidad de vida a un precio menor que en la capital — que toma el testigo de los primeros modelos de ciudad dormitorio basada en la construcción de polígonos de vivienda en los núcleos más cercanos a la ciudad central o en los más consolidados. Asimismo, la búsqueda de un estatus social, incentivado por la imagen de algunas urbanizaciones primigenias se materializa en urbanizaciones cerradas y autoexcluidas, tanto exentas como insertas en las tramas urbanas; este tipo de urbanizaciones son la quintaesencia de la oferta de unos valores tradicionales, ahora degradados y entendidos de una forma simple como meros productos de venta. Se evoluciona así pues muy negativamente hacia un modelo urbano muy agresivo con un recurso tan importante como el suelo, que ha sido ocupado muy desordenadamente y opacado en sus valores patrimoniales, ambientales y paisajísticos (Delgado-García, 2009).

\section{Figura 2}

\section{EVOLUCIÓN DEL TERRITORIO METROPOLITANO DE SEVILLA}

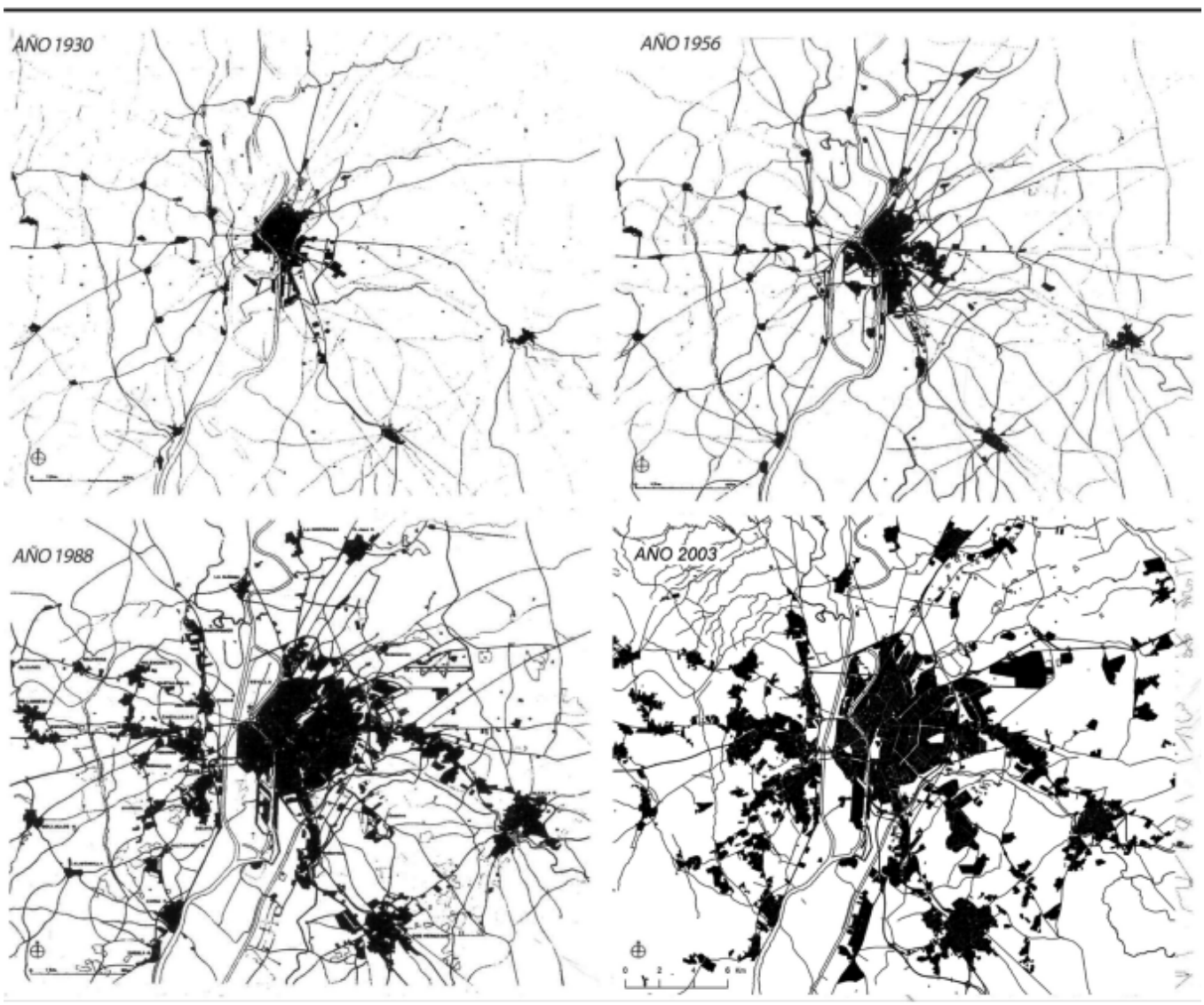

Fuente: Arias García, P. y Recuenco Aguado, L., 1999, adaptada por García, 2007

El origen de su proceso de conformación como espacio metropolitano se sitúa a principios de la década de los sesenta cuando, dentro de la política desarrollista y de apertura externa llevada a cabo en la España franquista, se aprueba para Sevilla el Polo de Desarrollo, que, en consonancia con lo ocurrido en otras áreas, contribuyó al crecimiento económico, aunque en 
menor medida de lo esperado, pero sobre todo supuso un cambio de escala de los procesos de urbanización. La amplia superficie de suelo para la implantación de actividades industriales que se delimitó en dos municipios de la parte suroriental de la primera corona (Alcalá de Guadaíra y Dos Hermanas), además de en la capital, resultó un estímulo para que se iniciase un lento proceso de metropolización que no llegó a consolidarse hasta mediados de los años ochenta.

Así las cosas, no puede extrañar que el primer documento que analiza en su conjunto la aglomeración incluyendo en ella 18 municipios date de 1984. Dos años más tarde, se aprobaron las Bases para la Coordinación Urbanística del Área Metropolitana y en 1989 las Directrices para la Coordinación Urbanística, que ampliaban el ámbito hasta incluir 20 municipios; hubo que esperar otros diez ańos para que se diseñase una propuesta de Plan de Ordenación del Territorio de la Aglomeración, que incluía ya 22 términos municipales. Por último, el Plan de Ordenación del Territorio de la Aglomeración, recientemente aprobado, amplía considerablemente su ámbito de actuación abarcando ahora un total de 46 municipios que se dividen en dos coronas: la primera compuesta por los 22 que conformaban ya antes la aglomeración y la segunda que incluye los 24 restantes (Consejería de Obras Públicas y Transportes. Junta de Andalucía, 2007) (Figura 3).

Sin entrar a valorar ahora detalladamente estas actuaciones, no cabe duda de que los procesos de ordenación territorial, siempre necesarios, adquieren especial importancia en los ámbitos metropolitanos, dada la complejidad de su funcionamiento y la imprescindible coordinación de su gestión. En el caso de Sevilla, el dilatado horizonte temporal y las sucesivas modificaciones de los documentos de planificación ponen de manifiesto que éste es un tema central. Asimismo, que son constantes las resistencias municipales ante la incorporación de un nuevo marco normativo y de gestión. 
Figura 3

\section{EVOLUCIÓN DE LA PROPUESTA DE ÁREA METROPOLITANA DE SEVILLA}

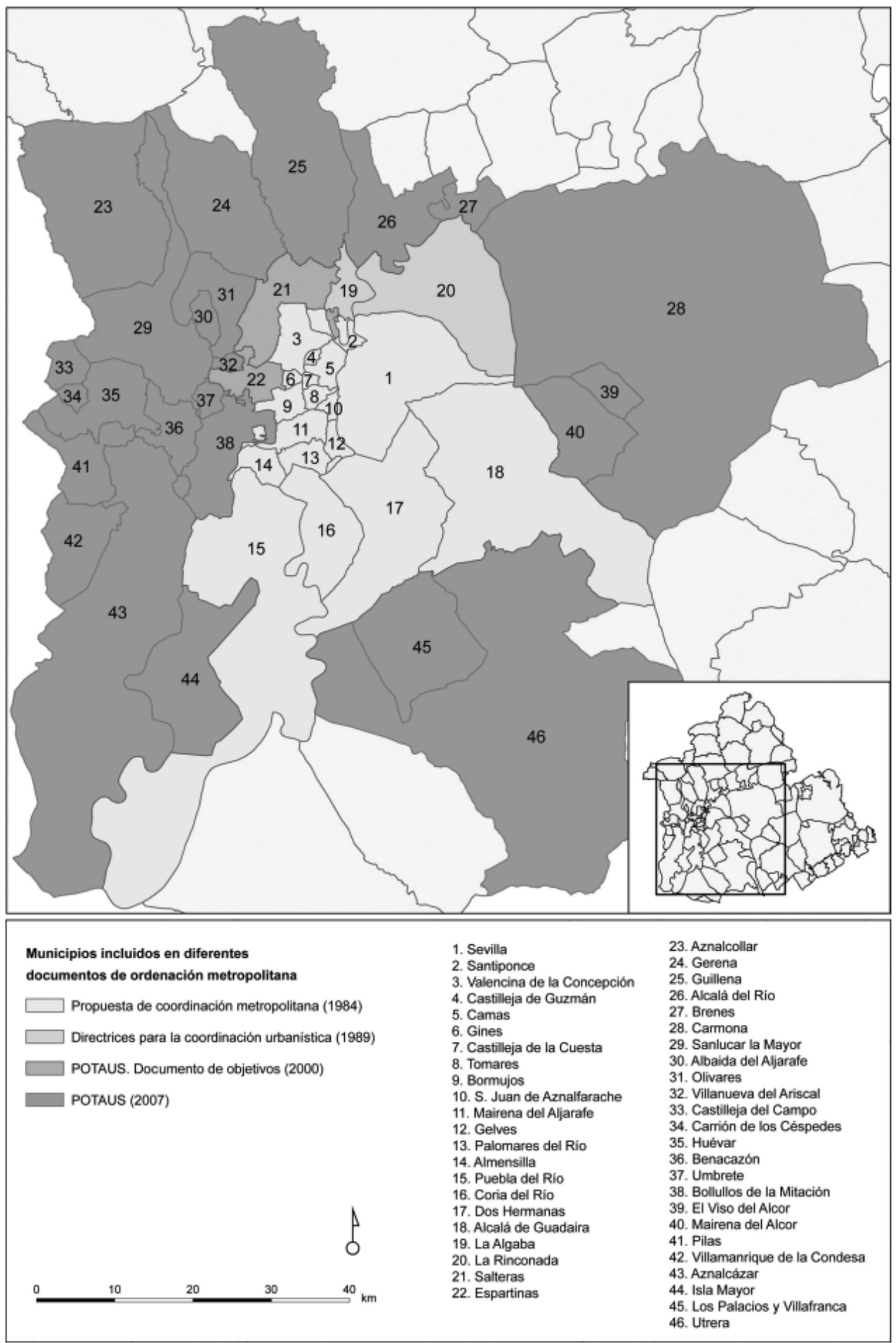

Fuente: Elaboración propia a partir de documentos de ordenación metropolitana 


\section{Evolución de la población de la aglomeración.}

La población existente en la aglomeración no ha experimentado un fuerte crecimiento durante los últimos años, pasando de un total de 1.243.866 habitantes en 1991 a 1.450.214 en 2007. De ellos, 1.158 .007 (lo que representa algo más del 80\%) se localiza en los 22 municipios que conforman la primera corona metropolitana junto a la ciudad central, la cual, aunque está perdiendo peso en el total, concentra en torno al 50\% de la población del área. Es por tanto la primera corona la que está absorbiendo hasta ahora la mayor parte del crecimiento, aunque dentro de ella existen diferencias territoriales significativas, pudiendo identificarse los siguientes tipos de municipios (Figura 4):

- Consolidados con un crecimiento gradual en los últimos decenios: Alcalá de Guadaíra y La Rinconada.

- Pioneros como ciudad dormitorio, estancados desde los setenta: Camas y San Juan de Aznalfarache.

- Con un intenso crecimiento, que llega a ser exponencial desde los setenta y sobre todo en los noventa: Bormujos, Castilleja de la Cuesta, Ginés, Mairena del Aljarafe y Tomares. Se incluyen también en este grupo otros municipios cuyo crecimiento ha sido más reciente: Almensilla, Castilleja de Guzmán, Espartinas, Palomares del Río y Valencina de la Concepción o Gelves. Especial importancia tiene Dos Hermanas que ha superado ya los 120.000 habitantes.

- Con menor crecimiento poblacional desde mediados del siglo XX, que se corresponden en general con los núcleos más alejados de los grandes ejes de acceso directo a la ciudad central y de las dinámicas de conurbación más consolidadas: Coria del Río, Puebla del Río, Santiponce, La Algaba, Salteras. 

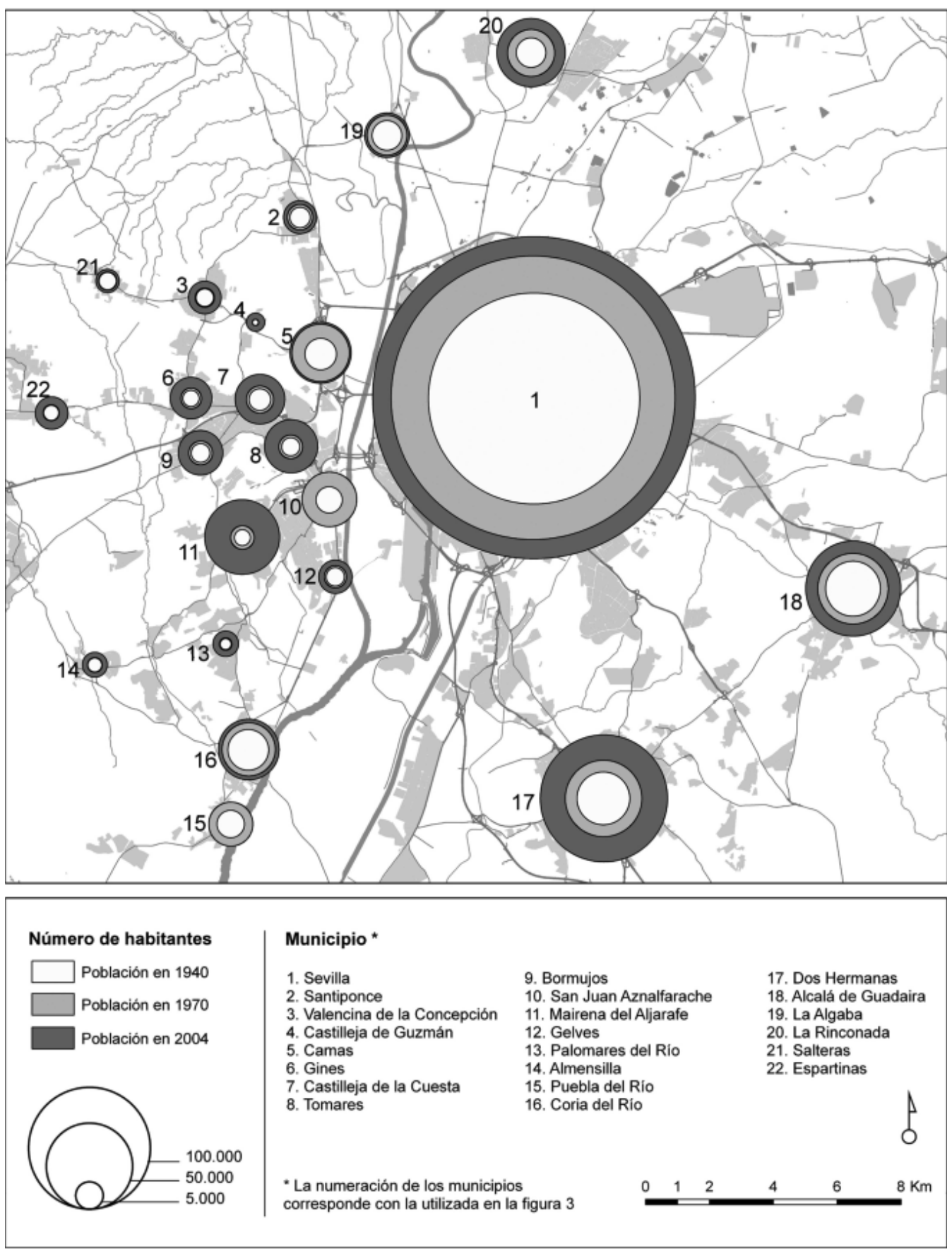

Fuente: García García, 2007

Si se analiza el comportamiento reciente de la población en el conjunto de la aglomeración, se observa que el porcentaje de crecimiento total de los municipios que conforman la segunda corona metropolitana es ya muy cercano a los de la primera (Cuadro 1). Esto pone de 
manifiesto la cada vez más evidente existencia de movimientos difusores de la población hacia municipios periféricos entre los que pueden diferenciarse los siguientes:

- En primer lugar, a los núcleos más alejados de Sevilla, aunque situados en la parte occidental de la primera corona metropolitana, que se caracterizan por haber experimentado un crecimiento exponencial en los últimos seis años: Almensilla, Castilleja de Guzmán, Espartinas, Palomares del Río y Salteras.

- En segundo lugar, a ciertos municipios de la segunda corona, entre los que destacan Mairena del Alcor al este, Los Palacios al sur y de nuevo, sobre todo, algunos de los situados al oeste: Albaida del Aljarafe, Bollullos de la Mitación y Umbrete.

EVOLUCIÓN DE LA POBLACIÓN EN EL CONJUNTO DE LA AGLOMERACIÓN

\begin{tabular}{lrrr}
\hline & Población 2007 & Población 2001 & $\begin{array}{c}\text { \% Crecimiento } \\
\text { 2001-2007 }\end{array}$ \\
\hline Sevilla ciudad & 699.145 & 684.633 & 2,12 \\
$1^{\text {a }}$ Corona metropolitana & 475.653 & 412.753 & 15,24 \\
$2^{\text {a }}$ Corona metropolitana & 275.416 & 247.444 & 11,3 \\
Total Área Metropolitana & $\mathbf{1 . 4 5 0 . 2 1 4}$ & $\mathbf{1 . 3 4 4 . 8 3 0}$ & $\mathbf{7 , 8 4}$ \\
\hline \multicolumn{4}{c}{ Fuente: Elaboración propia, a partir de Censo 2001 y la Revisión del Padrón, 2007 }
\end{tabular}

El incremento de la población de la aglomeración de Sevilla no resulta per se especialmente significativo desde la perspectiva de la inteligencia territorial, pero sí su distribución espacial y las lógicas a las que ésta responde. Así, en los últimos dos decenios se ha potenciado el modelo de la ciudad dormitorio metropolitana, genérica, de baja densidad, alto consumo de suelo y fundamentalmente ligada al sector de la construcción.

\section{Transformaciones económicas y nuevas tendencias urbanas}

Siendo ésta la mayor aglomeración urbana de Andalucía y Sevilla la capital administrativa de la Comunidad Autónoma, no puede extrañar que sea éste el ámbito funcional más importante de la región y el económicamente más dinámico.

Como muestra la figura 5, la mayor parte del empleo se concentra en Sevilla, que con un total de 345.635 supera con mucho a los municipios que le siguen en importancia, Dos Hermanas y Alcalá, cuyo empleo respectivo es de 39.561 y 29.275. Pese a lo apuntado, el crecimiento experimentado por el empleo en algunos de los municipios occidentales de la periferia metropolitana es significativo, tanto en los que forman parte de la primera corona (Bormujos o Gelves), como en los que conforman la segunda (Bollullos de la Mitación o Huévar del Aljarafe). 
Figura 5

\section{LOCALIZACIÓN Y EVOLUCIÓN RECIENTE DEL EMPLEO EN EL ÁREA} METROPOLITANA DE SEVILLA

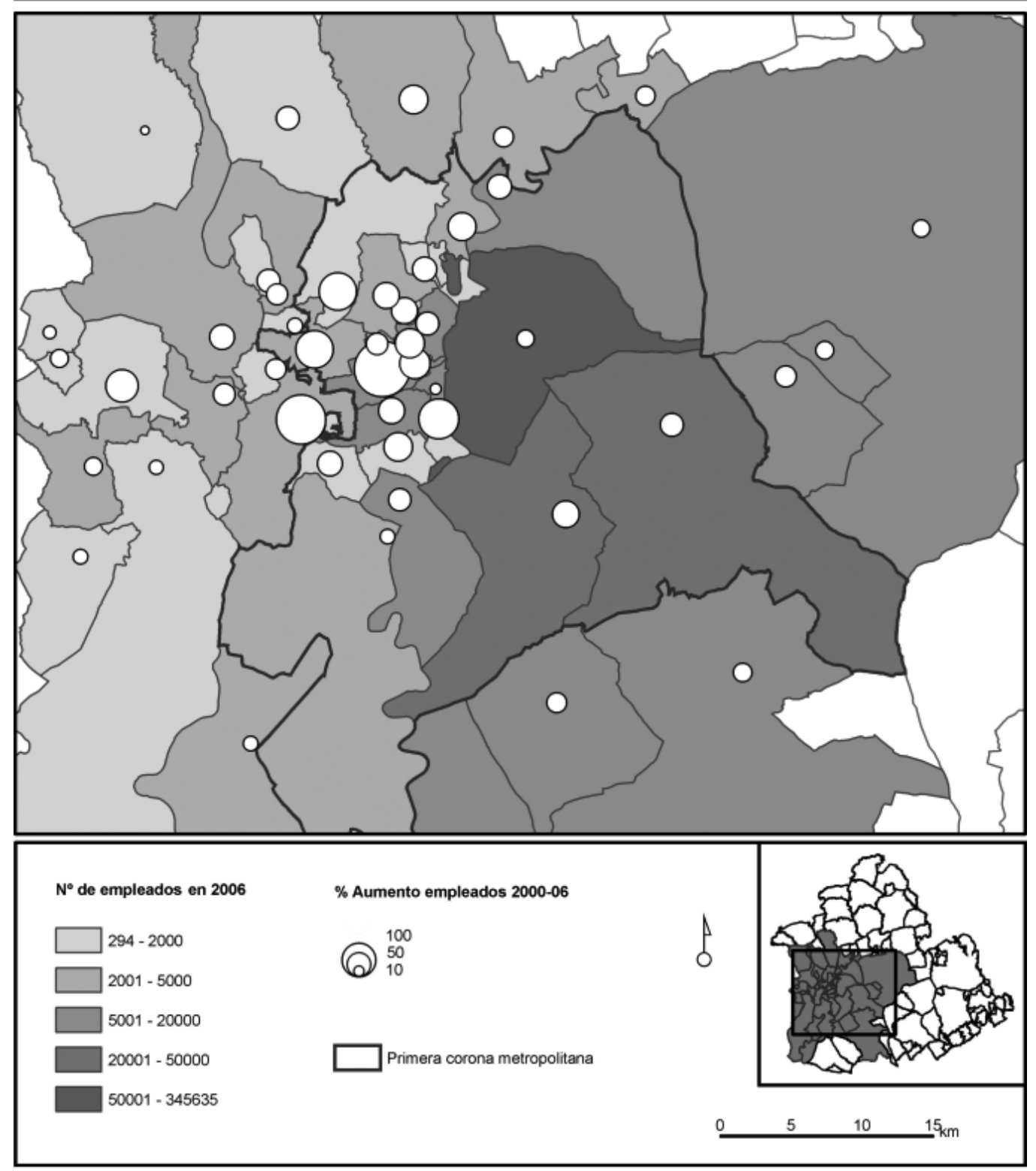

Fuente: Elaboración propia, a partir de datos de la Tesorería General de la Seguridad Social

Desde el punto de vista sectorial, la estructura económica de la aglomeración está fuertemente terciarizada, lo que hay que poner en relación con el rol que ejerce Sevilla como capital regional, con la consiguiente presencia de la administración pública y la existencia de un elevado número de empleos comerciales y de servicios avanzados relacionados con su centralidad funcional.

Las distintas actividades se distribuyen por el territorio según el sector de actividad en el que se integran, el tipo de empresas, la accesibilidad o el precio del suelo, aunque Sevilla cuenta 
con un mayor número de establecimientos, sobre todo del sector servicios, en el que concentra en torno al $70 \%$ del empleo de la aglomeración.

Por su parte, la industria, en consonancia con lo que ha sido hasta ahora la tónica general en Andalucía, tiene una presencia escasa, como lo muestra el hecho de que los establecimientos industriales del área no alcancen ni el 10\% de los que cuentan con actividad económica en la aglomeración, lo que sin duda es especialmente preocupante puesto que este ámbito concentra casi una cuarta parte de la industria andaluza. Esta actividad se encuentra más equilibradamente repartida por el territorio, de forma que Sevilla sólo concentra el $47 \%$ de los empleos, localizándose el 37\% en la primera corona en la que destacan especialmente por su importancia Alcalá de Guadaíra, Dos Hermanas y La Rinconada (Consejería de Obras Públicas y Transportes. Junta de Andalucía, 2006; Mendoza, 2006). Pese a lo señalado, se están observando recientemente nuevos movimientos difusores hacia la segunda corona metropolitana, que en ocasiones se extienden hacia espacios periurbanos.

El sector de la construcción ha sido especialmente dinámico durante los últimos años, convirtiéndose el mercado inmobiliario en el principal motor económico de buena parte del entorno metropolitano, sobre todo en el sector occidental y en los municipios de la primera corona, aunque más recientemente se ha extendido a otras muchas zonas del segundo anillo metropolitano. La expansión incontrolada de la construcción con un modelo de baja densidad ha propiciado un consumo voraz de suelo, cambios drásticos del paisaje y una redefinición sin precedentes de los modos de vida y la movilidad. Como señaló Feria Toribio (2000), resulta ilustrativo el hecho de que el suelo urbanizado del ámbito se haya multiplicado por 10 en las tres últimas décadas, mientras que su población ni siquiera lo ha hecho 1,5 veces.

Entre las transformaciones económicas ligadas al territorio metropolitano, destacan significativamente el incremento de los centros comerciales, que adquieren la fisonomía de grandes aglomeraciones de ocio y consumo y que se han convertido en referentes económicos y sociales en un área metropolitana funcionalmente fragmentada, pero donde los modos de vida de buena parte de la población tienden a la homogeneidad.

\section{La capacidad innovadora del territorio metropolitano}

Como es sabido, la globalización económica establece un duro sistema de competencia mundial, que obliga a las empresas y a los territorios a mantenerse en estado de alerta ante las nuevas oportunidades que puedan conferirles ventajas; no puede extrañar por ello que, como se señaló anteriormente, exista un acuerdo bastante generalizado en considerar que la actitud innovadora resulta una de las principales claves del éxito de las empresas y de los territorios (Aydalot-Keeble, 1988; Maillat-Kebir, 1998; Crevoisier-Camagni, 2000; Méndez, 2002 y 2007).

Para analizar la capacidad innovadora de la aglomeración metropolitana de Sevilla se han seleccionado tres de los indicadores al uso. Dichos indicadores están relacionados con los recursos para llevar a cabo las innovaciones, el esfuerzo realizado por las empresas para innovar y los resultados conseguidos con la innovación (Cuadro 2). 
Cuadro 2

ALGUNOS INDICADORES DE CAPACIDAD INNOVADORA DEL ÁREA METROPOLITANA DE SEVILLA

\begin{tabular}{|c|c|c|c|}
\hline & $\begin{array}{l}\text { Recursos para la } \\
\text { innovación }\end{array}$ & Esfuerzo innovador & $\begin{array}{l}\text { Resultado de la } \\
\text { innovación }\end{array}$ \\
\hline & $\begin{array}{l}\% \text { Pob. con estudios } \\
\text { superiores / Pob }> \\
16 \text { años (2001) }\end{array}$ & $\begin{array}{c}\mathrm{N}^{\circ} \text { empresas con } \\
\text { proyectos de I+D } \\
\text { financiados por el CDTI } \\
(2000-05)\end{array}$ & $\begin{array}{c}\mathrm{N}^{\circ} \text { de patentes } \\
\quad(2000-07)\end{array}$ \\
\hline Sevilla & 18,29 & 31 & 466 \\
\hline $1^{\text {a }}$ Corona metropolitana & 13,73 & 12 & 173 \\
\hline $2^{\mathrm{a}}$ Corona metropolitana & 6,14 & 3 & 26 \\
\hline Total Área Metropolitana & 9,91 & 46 & 665 \\
\hline
\end{tabular}

Fuente: Instituto Nacional de Estadística, Centro para el Desarrollo Tecnológico Industrial (CDTI) y Oficina Española de Patentes y Marcas

En primer lugar, resulta de interés considerar un recurso tan básico para la innovación como la población con preparación para realizar innovaciones. En este sentido, puede resultar útil el porcentaje de aquélla que cuenta con estudios universitarios respecto a la que tiene edad laboral. En relación a ello, resulta significativa la diferencia entre la ciudad central y el resto de los municipios que conforman la aglomeración, reduciéndose, además, el porcentaje de población con titulación superior en los municipios situados en la segunda corona.

Otro tanto ocurre si se utiliza como indicador del esfuerzo realizado para innovar medido por el número de empresas acogidas a proyectos de $\mathrm{I}+\mathrm{D}$ gestionados por el CDTI (Centro para el Desarrollo Tecnológico Industrial) entre los años 2000 y 2005; en este sentido, mientras son 31 las localizadas en Sevilla ciudad, sólo 12 de las situadas en la primera corona y 3 de las emplazadas en la segunda han recibido ayudas de dicho organismo, lo que pone en evidencia las diferencias territoriales existentes respecto al comportamiento empresarial.

La incidencia de la actividad innovadora en un territorio puede estimarse también a partir de los beneficios socioeconómicos de ella derivados, y éstos pueden ser medidos considerando los sistemas de protección aplicados a los nuevos conocimientos. El número de patentes solicitadas y concedidas puede ser así un buen indicador de la capacidad innovadora de un territorio, aunque hay que reconocer que su empleo presenta limitaciones asociadas a la existencia de otros medios para la protección de las invenciones y de la diferente propensión a patentar de los distintos sectores de actividad (Pavitt, 1984). Pese a ello, la patente es el instrumento más utilizado para la protección de los nuevos conocimientos, ya que con ella se concede al propietario el derecho de prohibir la explotación de un invento; de ese modo el titular de la patente dispone del monopolio territorial y temporal de explotación, con todo lo que ello supone de cara a la competitividad. De las 665 patentes realizadas por las empresas del área metropolitana de Sevilla entre los ańos 2000 y 2007, un 70,07\%, se localizan en la ciudad central, correspondiendo a las empresas de la primera corona un $26 \%$ y sólo un 3,93\% a las de la segunda. 
$\mathrm{Al}$ margen de lo limitadas que resultan las innovaciones en un ámbito claramente condicionado por una estructura económica caracterizada por el escaso peso de la industria y el reducido tamaño medio de las empresas en él localizadas, no puede olvidarse que se trata del territorio que cuenta con una buena parte de la población más cualificada y de las empresas vinculadas a los sectores más estratégicos, tanto industriales como de servicios, de toda Andalucía.

Obviamente, lo aquí analizado está relacionado con la visión más economicista de los territorios inteligentes, aunque tiene una gran incidencia en la capacidad competitiva y el dinamismo socioeconómico del ámbito. Dado lo que ello significa y lo importante que resulta para impulsar los procesos de desarrollo, no puede extrañar que las políticas públicas estén dedicando especial atención a todas aquellas medidas que puedan favorecer los comportamientos innovadores y, en ese sentido, en el Plan Andaluz de Investigación, Desarrollo e Innovación, de la Junta de Andalucía, se ha creado una serie de espacios y centros ligados a la innovación y al conocimiento, que tienen peso considerable en el área metropolitana: 3 parques tecnológicos-científicos, 2 parques de innovación empresarial, así como 7 centros tecnológicos de distinto tipo.

Por otra parte, y en consonancia con lo ocurrido en otros ámbitos, se pone en evidencia la marcada concentración territorial de la innovación en el área metropolitana de Sevilla. Un ejemplo ilustrativo resulta el hecho de que en la ciudad central se ubiquen uno de los parques científicos-tecnológicos, uno de los parques de innovación empresarial y cuatro de los centros tecnológicos.

Más difícil resulta, como contrapunto, analizar el comportamiento más o menos innovador desde la perspectiva socioinstitucional, dado que no existen indicadores al respecto, lo que hace necesario la generación de información complementaria, en buena parte de carácter cualitativo, que requiere la realización de estudios de caso.

\section{Un territorio con importantes recursos naturales y culturales}

La nueva forma de entender el territorio, a la que antes se hizo referencia, debe encuadrarse en el proceso de progresiva identificación e incorporación de recursos a las lógicas socioeconómicas, generalizándose la tesis de que todo proceso de desarrollo requiere la utilización imaginativa, racional, equilibrada y dinámica de todos los bienes patrimoniales (CEPAL, 1991 y 1992; Allende, 1995; Ortega Valcárcel, 1998 y 2004; Caravaca-González-Silva, 2005). Se considera así que es necesario poner en valor los recursos existentes en cada ámbito con objeto de que puedan constituir la base de su desarrollo.

Esto produce una segunda sinergia positiva, en tanto el respeto y la difusión de los hitos patrimoniales, los paisajes fundantes, o la variedad de usos característicos del territorio, resultan aspectos básicos para el reconocimiento individual y colectivo (García-Delgado-Ojeda, 2008). De este modo, parece que sólo deberían ser calificados como inteligentes aquellos territorios que, utilizando sus propios recursos de una forma ambiental, social y económicamente eficiente, consiguen generar verdaderos procesos de desarrollo territorial integrado. 
Dejando ahora al margen otros tipos de recursos, a algunos de los cuales ya se ha hecho referencia, se analiza aquí el patrimonio natural y cultural heredado que adquiere ahora una nueva dimensión.

El medio físico de la aglomeración metropolitana de Sevilla cuenta con algunos componentes de alto valor patrimonial. Destaca sobre todo por su importancia el río Guadalquivir, que no sólo es el elemento que conforma físicamente al área sino que es el eje que articula los flujos ecológicos de toda la zona, habiendo sido declarado su curso bajo Lugar de Importancia Comunitaria por la Unión Europea. No puede olvidarse, así mismo, que está incluida en la aglomeración una parte del Parque Natural de Doñana; se trata de una zona de transición entre el litoral y el curso bajo del Guadalquivir en la que confluyen distintos ecosistemas y que cuenta con un paisaje singular y una gran riqueza botánica y faunística.

Por su parte, el patrimonio cultural es especialmente rico en el ámbito, lo que está estrechamente relacionado con su privilegiada posición al lado del Guadalquivir que lo hizo atractivo para la localización de asentamientos humanos desde épocas prehistóricas. Ello explica el elevado número de bienes que gozan de alguna figura de protección ( 3 inmuebles declarados Patrimonio Mundial, 5 conjuntos históricos y 3 arqueológicos, 168 monumentos). Entre ellos cabe mencionar las ciudades romanas de Itálica en Santiponce y Orippo en Dos Hermanas, la necrópolis de Carmona, el yacimiento de El Carambolo en Camas, el complejo de dólmenes de Valencina de la Concepción y de Castilleja de Guzmán y el conjunto patrimonial de El Gandul en Alcalá de Guadaíra. Existen, además, más de 1.300 bienes incluidos en el Catálogo General del Patrimonio Histórico de Andalucía.

Como complemento a lo anterior, hay que tener también en cuenta que un buen número de cascos urbanos tienen un alto valor patrimonial, como es el caso de los conjuntos históricos de Sevilla, Carmona, Utrera, Olivares y Sanlúcar la Mayor.

Ahora bien, ante el reto de valorar la inteligencia con la que se gestiona este patrimonio, no cabe sino tener fundadas cautelas en el caso de la aglomeración de Sevilla. La situación de algunos de sus principales recursos patrimoniales da fe de ello.

Casos como el del yacimiento romano de Itálica, en Santiponce, evidencian una manifiesta incapacidad de la política local durante el último siglo para aprovechar económica y socialmente la singularidad del recurso. Frente a ello, todavía es un frente de conflicto entre sectores de la población que se sienten amenazados por cualquier ampliación de las intervenciones arqueológicas y otros que encuentran en las mismas un recurso para sus economías.

A su vez, el reconocimiento del valor patrimonial de algunos elementos o zonas no es óbice de que se reproduzcan otras tantas contradicciones. En el caso de los conjuntos históricos declarados, además de constantes problemas de transformación, terciarización, musealización o gentrificación, aparecen otras incongruencias, como ocurre por ejemplo en el Conjunto Histórico de Sevilla, donde, a pesar de la existencia de un Plan Especial de Protección aprobado en 1994, aún faltan por desarrollar algunas de sus propuestas. 


\section{Problemas y desórdenes asociados al mal uso de los recursos territoriales.}

En abierta contradicción con las tesis que abogan por la necesidad de utilizar racionalmente los propios recursos, el modelo de crecimiento urbano de la aglomeración metropolitana de Sevilla está basado en el aprovechamiento intensivo de aquéllos más genéricos sin tener para nada en cuenta los que le son específicos. Ello supone que, junto a la existencia de nuevas oportunidades locales también surgen problemas comunes en territorios diferentes, lo cual es sintomático de la simplificación de los procesos territoriales en el actual modelo metropolitano.

Siguiendo las tendencias generales de otras aglomeraciones, la ciudad de Sevilla crece mucho menos que el conjunto del ámbito, aunque ha reforzado su centralidad al concentrar buena parte de las llamadas funciones comando existentes no sólo en el área sino también en Andalucía (Magnaghi, 1981). Estos comportamientos, estrechamente relacionados con los procesos económicos y territoriales en curso, se han visto ampliados porque el interés por potenciar la competitividad de Sevilla a escala global ha contribuido a que se hayan dejado en un segundo plano aquellas actuaciones encaminadas a lograr una mayor cohesión territorial.

Como contrapunto a lo anterior, los procesos de difusión territorial hacia las coronas metropolitanas, tanto de la población como de algunas actividades económicas, han dado lugar a un crecimiento urbano extensivo muy alejado del modelo tradicional; proceso que no sólo resulta impactante por su extensión superficial o por la intensidad de los cambios que ha propiciado, sino también por la velocidad con la que éstos se están sucediendo. De este modo se evidencia un desorden, una confusión y una simplificación del territorio aún difícilmente digeribles por el ámbito metropolitano y sus habitantes, y que hasta el momento no ha encontrado el contexto normativo necesario para ser reglado, lo que es sin duda un claro símbolo de falta de inteligencia, es decir, de torpeza.

Surgen, pues, en el área metropolitana de Sevilla problemas y disfunciones de distintos signo, pero en una u otra medida directamente relacionados con el modelo con el que se ha configurado la aglomeración en los últimos decenios, y aunque son extensibles a todo su conjunto, en determinados ámbitos pueden ser especialmente intensos y preocupantes.

Por una parte, el despilfarro propio de una urbanización extensiva y tremendamente voraz, que supone el sellado de suelos, en algunos casos considerados de alta calidad agrícola, y la simplificación de sus usos. Esto tiene una clara materialización en el paisaje de la aglomeración, que sufre un fuerte deterioro, incluso en espacios considerados de alta calidad ambiental. Nos encontramos, en general, con el paisaje característico de muchas áreas metropolitanas, definido por su discontinuidad y la confusión de elementos y aprovechamientos: agrícolas, barbechos urbanos, infraestructuras, espacios comerciales, industriales y residenciales... y en el que, a una escala más inmediata, los escenarios urbanos se vuelven homogéneos y banales. Como consecuencia de todo ello se produce una pérdida de identidad y diversidad territorial que constituye un verdadero problema y que, obviamente, no puede considerarse comportamiento propio de un territorio inteligente. 
En este marco, los hitos patrimoniales naturales y culturales se encuentran en una situación de fragilidad y vulnerabilidad acuciante, pues la expansión de lo urbano, además de modificar drásticamente el paisaje, está afectando a la descontextualización de referentes. Desde esta situación se puede comprender cómo bienes ampliamente reconocidos se convierten incluso en elementos molestos para determinados grupos preferentemente ligados a la expansión inmobiliaria. Ante ello, la intervención pública para paliar la presión inmobiliaria acaba optando por su protección más radical, a expensas de la pérdida de su capacidad como espacio visitable y de reconocimiento que puede contribuir a mejorar la calidad de vida de los ciudadanos.

Pero, a su vez, este proceso urbanizador, en buena parte insuficientemente controlado, ha contribuido a potenciar algunos de los riesgos considerados "naturales". Por una parte, y como ya se ha señalado, el fuerte riesgo de inundación afecta a una significativa parte del área, aunque esté ya controlado en la ciudad central con diversas actuaciones de encauzamiento de los cursos fluviales. Por otra, junto a lo anterior, las pérdidas de suelo asociadas a la erosión en las laderas de los escarpes del Aljarafe y los Alcores generan riesgos de deslizamientos de terrenos, algunos de los cuales tienen un uso residencial.

Estos cambios en el territorio y en sus dinámicas tienen un fondo social igualmente relevante. Así, no puede dejarse al margen el problema que representa el incremento de la demanda de energía, en buena parte asociado a la adopción de un modelo de ciudad que menosprecia tanto en su arquitectura como en su urbanismo los requerimientos sociales y ambientales del ámbito territorial en el que se implanta. Sevilla y los núcleos de su área metropolitana funcionan como ciudades "globalizadas", en las que no tienen cabida los conocimientos secularmente adquiridos, por ejemplo de adaptación a las altas temperaturas estivales mediante un callejero compacto, anchos muros y tapiales, o patios interiores. Pero tampoco asume los presupuestos urbanísticos más racionalistas en relación a la circulación del viento o la insolación y ni mucho menos se hace eco de las tendencias más recientes sobre arquitectura bioclimática.

Este triunfante modelo urbanístico, marcado además por su dispersión, está estrechamente asociado a la proliferación de formas de urbanización y tipologías edificatorias en las que priman las viviendas unifamiliares, que, según señalan Magrinyá y Herce (2007), multiplican por 6 el consumo energético. Como consecuencia de todo lo anterior, el crecimiento de las infraestructuras viarias parece imparable, con un paralelo aumento del parque automovilístico, dado que la red de transportes públicos es absolutamente insuficiente. Es por eso que el modelo de movilidad está basado en el uso masivo del vehículo privado, convertido en un auténtico requisito vital para muchos de sus habitantes.

A ello hay que añadir, entre otras cosas, la creciente generación de residuos, desechos y vertidos cuyos costes de recogida y tratamiento se elevan considerablemente cuanto más dispersas sean las formas de urbanización; lo que da como resultado que la huella ecológica de la aglomeración sea 54,5 veces superior a la superficie que ocupa (Cano Orellana, 2004).

Siendo graves los problemas hasta ahora comentados, no hay que olvidar, además, que la relación entre el modelo urbano y territorial y los habitantes del área metropolitana tiene una repercusión aún más radical: el paso del papel de ciudadano al de consumidor. En efecto, 
la generación de espacios de competencia y de aprovechamiento intensivo ha conllevado una tendencia a modelos de comportamiento y de construcción basados en la primacía de lo privado sobre lo público, que se refrenda en nuevas formas y lugares de ocio y comercio, pero también en nuevos modos de entender los espacios públicos. Así los espacios de relación cotidiana o los paisajes identitarios, aparecen a menudo simplificados en sus contenidos y prostituidos en su imagen, mientras son sustituidos por espacios verdes, no exentos de interés, pero que a menudo quedan segregados de los usos y dinámicas más cotidianas de las tramas urbanas próximas (García García, 2007). Pese a ello, algunos de los espacios públicos de la aglomeración siguen siendo centrales en la memoria colectiva local y metropolitana, además de un referente para la conservación patrimonial, como es el caso, por ejemplo, del parque de Oromana en Alcalá de Guadaira.

Esta suma de comportamientos, todos ellos relacionados con un inadecuado uso de los recursos, pone en evidencia que el área no evoluciona positivamente en su proceso de desarrollo territorial, sino que, por el contrario, está comportándose de forma poco innovadora y menos aún inteligente, puesto que no está siendo capaz de contribuir a hacer mejores y más felices a sus ciudadanos.

\section{La aglomeración metropolitana de Sevilla: ¿̨territorio inteligente?}

Llegado a este punto, resulta obvio que la aglomeración metropolitana de Sevilla no puede ser considerada territorio inteligente desde ninguna de las dos perspectivas con las que se utiliza este concepto. No obstante, son más numerosos los comportamientos de instituciones y agentes públicos y privados que intentan avanzar en los procesos innovadores más vinculados al funcionamiento económico y, en consecuencia, la lectura de la evolución del ámbito desde esta perspectiva es mucho más positiva y esperanzadora, aunque aún no se haya avanzado significativamente en esta dirección.

Por el contrario, la evolución experimentada por la aglomeración respecto al uso de buena parte de sus recursos no sólo no va encaminada a resolver las disfunciones y problemas para así poder avanzar en el proceso de desarrollo, sino que está contribuyendo a agravar los existentes e incluso a generar otros nuevos.

Los ciudadanos han perdido protagonismo efectivo en la producción de su propio hábitat, que queda en manos de los “técnicos". A su vez, el interés por integrarse con una buena posición en el espacio global de las redes, ha hecho a los poderes públicos anteponer criterios de mercado en el proceso de producción de las ciudades, dedicando cada vez menos atención a la mejora de la calidad de vida y a favorecer el desarrollo personal de todos sus habitantes.

Esto hace urgente una redefinición de los modelos territoriales y urbanos, con una especial trascendencia en un contexto normativo supramunicipal, hasta el momento muy débil en la aglomeración. Además adquiere mayor significación en la medida que se refrende la expansión de las lógicas metropolitanas actuales a un segundo anillo en el caso de Sevilla. 
Pero no hay que olvidar que, además de la gestión inteligente del territorio, es fundamental su uso inteligente por parte de quienes lo viven y animan. En el caso de Sevilla es de obligada referencia el surgimiento de una red creciente de colectivos ciudadanos en el entorno metropolitano, que distan del perfil tradicional de movimientos convencionales ligados a situaciones de marginación social y económica, e inciden en la necesidad imperiosa de planear un nuevo orden territorial y de la puesta en valor de las idiosincrasias locales. Paradójicamente, no siempre cuentan con una conexión fluida con las redes vecinales de cada núcleo, debido al carácter supramunicipal de sus iniciativas y al hecho de que estos colectivos estén mayoritariamente formados por nuevos residentes. Más allá de los matices que puedan ser considerados en relación a estos movimientos, resultan muy importantes no sólo por su acción contestataria, sino porque ponen de manifiesto que él área metropolitana de Sevilla no sólo evoluciona en consonancia con las mayores aglomeraciones de su entorno, sino que también está comenzando a madurar como espacio ciudadano, con sus propias características.

Llegado este punto, parece de interés la participación en el debate sobre territorios inteligentes, utilizando para ello como ejemplo lo sucedido en ámbitos concretos, en este caso la aglomeración metropolitana de Sevilla.

\section{Referencias bibliográficas}

Albertos, J.M.; Caravaca, I.; Méndez, R. \& Sánchez, J.L. (2004). Desarrollo territorial y procesos de innovación socioeconómica en sistemas productivos locales. En J.L. Alonso et al. (Ed.). Recursos territoriales y geografía de la innovación industrial en España (pp. 15 60). Salamanca: Ediciones Universidad de Salamanca.

Allende, J. (1995). Desarrollo sostenible. De lo global a lo local. Ciudady Territorio. Estudios Territoriales, 104, 287-282.

Aguilar, A.G. (Coord.) (2006). Las grandes ciudades y su periferia regional. Experiencias en Latinoamérica y España. México DF: Cámara de Diputados-Universidad Nacional Autónoma de México.

Arias García, P. \& Recuenco Aguado, L. (1999). Consideraciones sobre la nueva ciudad-Sevillaante la revisión de su planeamiento. Sevilla: Universidad de Sevilla.

Ascelrad, H. (1999). Sustentabilidad y ciudad. EURE, 25, 74, 35-46.

Aydalot, Ph. \& Keebled, D. (1988). High technology industry and innovative environments. Londres: Croom Helm.

Benko, G. \& Lipietz, A. (Eds.) (1994). Las regiones que ganan. Distritos y redes. Los nuevos paradigmas de la geografía económica. Valencia: Institución Alfonso el Magnánimo.

Borja, J. (2007). Revolución y contrarrevolución en la ciudad global: las expectativas frustradas por la globalización en nuestras ciudades. EURE, 33, 100, 35-50.

Cano Orellana, A. (2004). Economía y sostenibilidad en las grandes regiones urbanas. Aproximación al cálculo de la huella ecológica de Sevilla y su área metropolitana. Sevilla: Sevilla Global S.A.M.

Caravaca, I. (1998). Los nuevos espacios ganadores y emergentes. EURE, 24, 73, 5-30.

Caravaca, I. (2006). La nueva industria urbana y metropolitana: procesos, estrategias y resultados. En P. Méndez, Industria y ciudad en España: nuevas realidades, nuevos retos (pp. 29-50). Madrid: Thomson Cívitas. 
Caravaca, I. (2007). El transfondo socioeconómico y ambiental de los espacios urbanos. En Artigues et al. (Eds.), Los procesos urbanos postfordistas (pp. 451-477). Palma de Mallorca: Grupo de Geografía Urbana (AGE).

Caravaca. I. \& Méndez, R. (2003). Trayectorias industriales metropolitanas. Nuevos procesos, nuevos contrastes. EURE, 29, 87, 37-50.

Caravaca, I.; González, G. \& Silva, R. (2005). Innovación, redes, recursos patrimoniales y desarrollo territorial. EURE, 31, 94, 5-24.

Carmona, M.; Heath, T.; Oc, T. \& Tiesdell, S. (2003). Public places, urban spaces. The dimensions of urban design. Oxford: Architectural Press.

Castells, M. (1990). Estrategias de desarrollo metropolitano en las grandes ciudades españolas: articulación entre crecimiento económico y calidad de vida. En J. Borja, et al., Las grandes ciudades en la década de los noventa (pp. 16-64). Madrid: Sistema.

Cating, M.; Lacour, C. \& Lung, Y. (2001). Innovation et développement regional. Revue d'Economie Régionale et Urbaine, 1, 3-20.

Comisión Económica para América Latina y el Caribe, CEPAL (1991). El desarrollo sustentable: transformación productiva, equidad y medio ambiente. Santiago de Chile: CEPALONU.

Comisión Económica para América Latina y el Caribe, CEPAL (1992). Equidad y transformación productiva: un enfoque integrado. Santiago de Chile: CEPAL-ONU.

Consejería de Obras Públicas y Transportes (2007). Plan de ordenación del territorio de la aglomeración urbana de Sevilla. Sevilla: Junta de Andalucía.

Delgado, B. \& García, A. (2009). Una aproximación a los nuevos paisajes de la metápolis en Andalucía. Scripta Nova, en prensa.

Feldman, M. (1994). The geography of innovation. Dordrecht: Kluwer.

Feria Toribio, J.M. (2000): La emergencia del territorio metropolitano de Sevilla. Diagnóstico general y conclusiones. Documentos preliminares para la elaboración del Plan General de Ordenación Urbana de Sevilla. Gerencia de Urbanismo del Ayuntamiento de Sevilla.

Feria Toribio, J.M. (2004). Problemas de definición de las áreas metropolitanas en España. Boletín de la Asociación de Geógrafos Españoles, 39, 85-99.

Fernández Durán, R. (1993). La explosión del desorden. La metrópoli como espacio de la crisis global. Madrid: Fundamentos.

Florida, R. (1995). Towards the learnings regions. Futures, 27, 5, 527-536.

Font, A. (2007). Morfologías urbanas contemporáneas de la baja densidad. En F. Indovina (Coord.), La ciudad de baja densidad. Lógicas, gestión y contención (pp. 97-108). Barcelona: Diputació de Barcelona.

García García, A. (2007). Los espacios públicos de Sevilla y su entorno metropolitano. Tesis Doctoral.

García García, A., Ojeda Ribera, J. \& Torres Gutiérrez, F.J. (2008). Hacia una nueva lectura de las ciudades y sus espacios: ausencias y emergencias en la ciudad inteligente. En A. García (Coord.), Espacios públicos, ciudad y conjunto histórico. Sevilla, IAPH.

García García, A.; Delgado Bujalance, B. \& Ojeda Ribera, J. (2008). Los paisajes simbólicos de la ciudad de Sevilla. ERÍA. Revista Cuatrimestral de Geografía, 73-74, 291-310.

Henry, G. (2007). Los costes económicos y sociales de la ciudad de baja densidad. En F. Indovina (Coord.), La ciudad de baja densidad. Lógicas, gestión y contención (pp. 203-242). Barcelona: Diputació de Barcelona. 
Indovina, F. (Coord.) (2007). La ciudad de baja densidad. Lógicas, gestión y contención. Barcelona: Diputació de Barcelona.

Jambes, J.P. (2001). Territoires apprenants. Esquisses pour le développement local du XXI siécle. París: L'Harmattan.

Konminos, N. (2002). Intelligent cities: innovation, knowledge systems and digital spaces. Londres: Sponpress.

Magrinyá, F. \& Herce, M. (2007). Los costes ambientales de la ciudad de baja densidad. En F. Indovina (Coord.), La ciudad de baja densidad. Lógicas, gestión y contención (pp. 243264). Barcelona: Diputació de Barcelona.

Magnaghi, A. (1981). Il sistema di goberno della regioni metropolitane. Milán: Franco Angeli. Maillat, D. \& Kebir, L. (1998). Learning region et systèmes territoriaux de production. Working Paper IRER, 9802a. Neuchâtel: Université de Neuchâtel.

Marina, J.A. (1993). Teoría de la inteligencia creadora. Madrid: Anagrama.

Martínez Fernández, M.C. (2004). La capacidad innovadora de las redes de desarrollo regional: El valor ańadido de la colaboración, la competitividad y la difusión del conocimiento. Información Comercial Española, 812, 55-69.

Mckimmon, D.; Cumbers, A. \& Chapman, D. (2002). Learning, innovation and regional development: a critical appraisal of recent debates. Progress in Human Geography, 26, 293-311.

Méndez, R. (2001). Transformaciones económicas y reorganización territorial en la región metropolitana de Madrid. EURE, 27, 80, 141-162.

Méndez, R. (2002). Innovación y desarrollo territorial: Algunos debates teóricos recientes. EURE, 28, 84, 63-84.

Méndez, R. (2007). El territorio de las nuevas economías metropolitanas. EURE, 33, 100, 51-67.

Méndez, R. (2008). Conocimiento e innovación para el desarrollo de ciudades intermedias. Ponencia presentada en el IX Coloquio de Geografía Urbana (AGE) (en proceso de publicación).

Méndez, R. \& Rodríguez Moya, J. (2007). Transformaciones productivas y nuevas formas urbanas: difusión de las actividades económicas en la región metropolitana funcional de Madrid. Anales de Geografía, 27, 2, 105-134.

Mendoza, A. (2006). Situación y tendencias de la industria en la aglomeración metropolitana de Sevilla. En Méndez-Pascual. Industria y ciudad en España: nuevas realidades, nuevos retos (pp. 213-241). Madrid: Cívitas.

Moulaert, F. \& Sekia, F. (2003). Territorial innovation models: a critical survey. Regional Studies, 37, 289-302.

Morgan, K. (1997). The learning region: institutions, innovation and regional renewal. Regional Studies, 31, 5, 491-503.

Nel-lo, O. (2007). Contra la dispersión, intensidad. Contra la segregación, ciudad. En F. Indovina (Coord.), La ciudad de baja densidad. Lógicas, gestión y contención (pp. 499-524). Barcelona: Diputació de Barcelona.

Ortega Valcárcel, J. (1998). El patrimonio territorial como recurso cultural y económico. Ciudades 4. Territorio y Patrimonio. Valladolid: Instituto de Urbanística de la Universidad de Valladolid. 
Ortega Valcárcel, J. (2004). La geografía para el siglo XXI. En J. Romero (Coord.), Geografía Humana (pp. 25-53). Barcelona: Ariel.

Pavitt, K. (1984). Sectorial patterns of technical change: towards a taxonomy and a theory. Research Policy, 13, 343-373.

Veltz, P. (1996). Mondialisation villes et territoires. L'economie d'archipel. París: P.U.F. 